\title{
ASYMPTOTIC EXPANSIONS FOR THE HEAT CONTENT
}

\section{S. DESJARDins}

Let $M$ be a compact Riemannian manifold with smooth boundary $\partial M$. We study the asymptotic expansions associated with the generalized heat operator $Q e^{-t P_{\mathcal{B}}}$ with suitable boundary conditions. A new invariant defined on the boundary of $M$ is introduced, and a method is given that relates the heat content asymptotics for the generalized heat operator and the standard heat operator $e^{-t P_{\mathcal{B}}}$ with the new boundary asymptotics. As an application, we compute the boundary asymptotics associated with an operator of Laplace type, and the asymptotics for a generalized operator constructed from an operator of Dirac type.

\section{Introduction.}

Many interesting results in global analysis have been obtained from the study of spectral invariants of the heat equation. The classical invariants are defined by the asymptotic expansion as $t \rightarrow 0^{+}$of

$$
\operatorname{Tr}_{L^{2}}\left(e^{-t \Delta}\right)=\sum_{\nu} e^{-t \lambda_{\nu}} \simeq \sum_{n=0}^{\infty} a_{n} t^{(n-m) / 2}
$$

where $e^{-t \Delta}$ is the fundamental solution of the heat equation

$$
\Delta+\partial_{t}=0
$$

and $\left\{\lambda_{\nu}\right\}$ the spectrum of the Laplace operator $\Delta$. While the spectrum depends on the global behaviour of $\Delta$, the asymptotics can be computed by integrating a local formula in the jets of the total symbol of the operator $\Delta$. The coefficients in this expansion are spectral invariants in the sense that if two manifolds have the same spectrum, then their asymptotic expansion must be equivalent.

Recently, new invariants have been computed. See [BD], [BG], and [DG] for examples. They are known as the heat content. The coefficients of the short-term asymptotic expansion for the heat content have direct applications in theoretical physics [McA]. While their study is parallel in many ways to the study of the classical invariants, their computation often requires 
modifications of the original techniques and, at times, new approaches. In this paper, we study the heat content associated with generalized heat operators. Our focus is on the functorial aspect of the problem rather than the actual lengthy computations.

Let $M$ be a compact Riemannian manifold of dimension $m$ with smooth boundary $\partial M$. Let $V$ be a vector bundle over $M$ and let $P: C^{\infty}(V) \rightarrow$ $C^{\infty}(V)$ be an elliptic partial differential operator of order $d>0$. Let $\mathcal{B}$ be a boundary operator, and denote by $P_{\mathcal{B}}$ the operator satisfying the boundary conditions determined by $\mathcal{B} f=0$. We assume the pair $\{P, \mathcal{B}\}$ satisfies a suitable ellipticity condition. Let $f_{1} \in C^{\infty}(V), f_{2} \in C^{\infty}\left(V^{*}\right)$, and denote by $\langle\cdot, \cdot\rangle$ the natural pairing between the bundle $V$ and the dual bundle $V^{*}$. Let $d x$ denote the Riemannian measure on $M$. We define the heat content as follows:

$$
\beta\left(f_{1}, f_{2}, P, \mathcal{B}\right)(t):=\int_{M}\left\langle e^{-t P_{\mathcal{B}}} f_{1}, f_{2}\right\rangle d x .
$$

Standard elliptic methods $[\mathbf{G r}]$ show that there exists an asymptotic series as $t \rightarrow 0^{+}$of the form

$$
\beta\left(f_{1}, f_{2}, P, \mathcal{B}\right)(t) \simeq \sum_{n=0}^{\infty} \beta_{n}\left(f_{1}, f_{2}, P, \mathcal{B}\right) t^{n / d} .
$$

The asymptotics are built universally and polynomially from the jets of the total symbols of $\{P, \mathcal{B}\}$ and the covariant derivatives of the $f_{i}$ 's. They contain information on the geometry of these operators, and on that of the underlying manifold and its boundary.

The definition of the heat content can be generalized as follows. Let $Q$ be an auxiliary differential operator of order $q>0$. We define the heat content corresponding to the operator $Q e^{-t P_{\mathcal{B}}}$ by

$$
\beta\left(f_{1}, f_{2}, Q, P, \mathcal{B}\right)(t):=\int_{M}\left\langle Q e^{-t P_{\mathcal{B}}} f_{1}, f_{2}\right\rangle d x .
$$

We say that the pair $\{P, \mathcal{B}\}$ is admissible whenever the following asymptotic expansion exists for $t \rightarrow 0^{+}$:

$$
\beta\left(f_{1}, f_{2}, Q, P, \mathcal{B}\right)(t) \simeq \sum_{n=0}^{\infty} \beta_{n}\left(f_{1}, f_{2}, Q, P, \mathcal{B}\right) t^{\frac{n-q}{d}}
$$

The generalized heat operator includes some interesting cases, many of which have been studied previously for the trace invariant.

Fegan and Gilkey $[\mathbf{F G}]$ have shown that, when $\partial M=\emptyset$, the trace invariants associated with the generalized heat operator $P^{a} e^{-t P^{b}}$, for some $a, b>0$, are characterized by the trace invariants of the operator $P$. That 
is, the asymptotics for this family of invariants can be written as linear combinations of the original asymptotics. In their paper, Fegan and Gilkey used the fact that when $P_{\mathcal{B}}$ is self-adjoint, the solution of the heat equation can be expressed in terms of the spectral data. This gives a relation between the trace of the heat kernel and the residues of the zeta function [MP]. There exists a similar link between the heat content asymptotics and a suitably modified zeta function. The analysis of $[\mathbf{F G}]$ can therefore be extended to obtain an analogous result for the heat content asymptotics on a manifold with non-empty boundary. This construction is valuable here since it allows us to exchange some of the complicated terms that arise in our formulas with expressions involving simpler invariants.

When the generalized heat operator is constructed from an operator of Dirac type $Q$, it is relatively easy to show that both the trace and the heat content asymptotics can be recovered from those of an operator of Laplace type $P=Q^{2}$ by means of a variational formula ([BG], $[\mathbf{D}]$ ). In this special case, the asymptotic expansions for the trace of $Q e^{-t P}$ have previously been studied in $[\mathbf{B G}]$; the corresponding trace invariants are important in index theory [APS]. In comparison, the computations required to obtain the heat content asymptotics from the variational formula are rather long and tedious, and the resulting expressions unwieldy; the terms that describe the asymptotics are not independent. Moreover, the application is limited in both case to operators of Dirac type.

To address this situation, we propose a functorial approach that is remarkably simpler and readily applicable to a wider class of operators. The novelty of our approach is that it involves a new invariant defined on the boundary of $M$. Aside from its technical merits, this new boundary invariant is especially interesting, for it yields an asymptotic expansion that did not arise previously in the study of the classical heat invariant. The methods described here should extend to the heat trace asymptotics. We hope that this will eventually lead to some new and interesting results.

This paper is arranged as follows. In Section 2, we use spectral theory to express the heat content in terms of the spectral resolution of the operator $P_{\mathcal{B}}$, and relate the asymptotics with a suitably modified zeta function. We then show how the Mellin transform and the properties of the zeta function can be used to obtain the heat content asymptotics for a generalized heat operator when $Q$ and $P$ are both powers of the same operator. In Section 3 , we introduce the heat content on the boundary, and develop some of its functorial properties. We also compute the asymptotics for the boundary invariant when $P_{\mathcal{B}}$ is an operator of Laplace type with Dirichlet and Neumann boundary conditions. In the last section, we use the results of Section 3 to compute the generalized heat content asymptotics for an operator of Dirac 
type.

\section{The zeta function for the heat content.}

We assume that $P_{\mathcal{B}}$ is self-adjoint and that $\{P, \mathcal{B}\}$ is admissible throughout this section. We use the fiber metric to identify $V$ and $V^{*}$. Let $\left\{\phi_{\nu}, \lambda_{\nu}\right\}$ denote the spectral resolution for $P_{\mathcal{B}}$, and let

$$
c_{\nu}(f)=\int_{M}\langle f(x), \bar{\phi}(x)\rangle d x
$$

be the Fourier coefficients. Then

$$
\beta\left(f_{1}, f_{2}, P, \mathcal{B}\right)(t)=\sum_{\nu} c_{\nu}\left(f_{1}\right) \bar{c}_{\nu}\left(f_{2}\right) e^{-t \lambda_{\nu}} .
$$

When $P_{\mathcal{B}}$ is positive definite, we can define the zeta function for the heat content of the operator $P_{\mathcal{B}}$ by

$$
\zeta\left(f_{1}, f_{2}, P_{\mathcal{B}}\right)(s):=\int_{M}\left\langle P_{\mathcal{B}}^{-s} f_{1}, f_{2}\right\rangle d x
$$

for $\mathbb{R e}(s)>0$, and use the Mellin transform to relate the zeta function and heat content asymptotics. Let $\Gamma$ be the classical gamma function defined by

$$
\Gamma(s):=\int_{0}^{\infty} t^{s-1} e^{-t} d t
$$

for $\mathbb{R e}(s)>0$. Let $\{P, \mathcal{B}\}$ be admissible, and let $P_{\mathcal{B}}$ be a self-adjoint positive definite operator of order $d$. Then the Mellin transform for $\beta\left(f_{1}, f_{2}, P, \mathcal{B}\right)$ is given by

$$
\Gamma(s) \zeta\left(f_{1}, f_{2}, P_{\mathcal{B}}\right)(s)=\int_{0}^{\infty} t^{s-1} \beta\left(f_{1}, f_{2}, P, \mathcal{B}\right)(t) d t .
$$

It has a meromorphic extension to $\mathbb{C}$ with isolated poles at $s=-n / d$ and

$$
\beta_{n}\left(f_{1}, f_{2}, P, \mathcal{B}\right)=\operatorname{Res}_{s=-\frac{n}{d}} \Gamma(s) \zeta\left(f_{1}, f_{2}, P_{\mathcal{B}}\right)(s) .
$$

This formalism will be most useful to us once it is extended to higher order operators obtained by taking powers of $P$. Assume that the pair $\left\{P^{b}, \mathcal{B}^{b}\right\}$ is admissible for

$$
\mathcal{B}^{b} f:=\mathcal{B} f \oplus \mathcal{B}(P f) \oplus \cdots \oplus \mathcal{B}\left(P^{b-1} f\right) .
$$

This condition is satisfied, for example, if $P$ is an operator of Laplace type and the operator $\mathcal{B}$ determines mixed boundary conditions. Although this is the case of our primary interest, we prove a more general case. Define

$$
\beta_{a, b}\left(f_{1}, f_{2}, P, \mathcal{B}\right)(t):=\int_{M}\left\langle P^{a} e^{-t P_{\mathcal{B}}^{b}} f_{1}, f_{2}\right\rangle d x .
$$


There exists an asymptotic expansion as $t \rightarrow 0^{+}$of the form

$$
\beta_{a, b}\left(f_{1}, f_{2}, P, \mathcal{B}\right)(t) \simeq \sum_{n=0}^{\infty} \beta_{n, a, b}\left(f_{1}, f_{2}, P, \mathcal{B}\right) t^{\frac{n-a d}{b d}}
$$

If $P_{\mathcal{B}}$ is a positive definite operator, we define the zeta function associated with $\beta_{a, b}\left(f_{1}, f_{2}, P, \mathcal{B}\right)$ by

$$
\zeta_{a, b}\left(f_{1}, f_{2}, P_{\mathcal{B}}\right)(s):=\int_{M}\left\langle P_{\mathcal{B}}^{-(b s-a)} f_{1}, f_{2}\right\rangle d x .
$$

These general invariants have been studied previously [D]. The next result shows that they can be expressed as a universal linear combination of simpler invariants.

Lemma 2.1. We adopt the notation established above:

(i) If $P_{\mathcal{B}}$ is positive definite,

$$
\Gamma(s) \zeta_{a, b}\left(f_{1}, f_{2}, P_{\mathcal{B}}\right)(s)=\int_{0}^{\infty} t^{s-1} \beta_{a, b}\left(f_{1}, f_{2}, P, \mathcal{B}\right)(t) d t .
$$

It has a meromorphic extension on $\mathbb{C}$ with isolated poles at $s=-\frac{n-a d}{b d}$, and

$$
\beta_{n, a, b}\left(f_{1}, f_{2}, P, \mathcal{B}\right)=\operatorname{Res}_{s=-\frac{n-a d}{b d}} \Gamma(s) \zeta\left(f_{1}, f_{2}, P_{\mathcal{B}}\right)(b s-a) .
$$

(ii) Let $C_{n, d, a, b}:=\lim _{s \rightarrow-\frac{n}{d}} b^{-1}\left\{\Gamma(s)^{-1} \Gamma\left(\frac{s+a}{b}\right)\right\}$. Then

$$
\beta_{n, a, b}\left(f_{1}, f_{2}, P, \mathcal{B}\right)=C_{n, d, a, b} \beta_{n}\left(f_{1}, f_{2}, P, \mathcal{B}\right)
$$

Proof. We proceed formally to write

$$
\begin{aligned}
\beta_{a, b}\left(f_{1}, f_{2}, P, \mathcal{B}\right)(t) & =\int_{M}\left\langle P^{a} e^{-t P_{\mathcal{B}}^{b}} f_{1}, \bar{f}_{2}\right\rangle d x \\
& =\int_{M}\left\langle P^{a} \sum_{\nu} c_{\nu}\left(f_{1}\right) e^{-t \lambda_{\nu}^{b}} \phi_{\nu}, \bar{f}_{2}\right\rangle d x \\
& =\sum_{\nu} c_{\nu}\left(f_{1}\right) \lambda_{\nu}^{a} e^{-t \lambda_{\nu}^{b}} \int_{M}\left\langle\phi_{\nu}, \bar{f}_{2}\right\rangle d x \\
& =\sum_{\nu} c_{\nu}\left(f_{1}\right) \bar{c}_{\nu}\left(f_{2}\right) \lambda_{\nu}^{a} e^{-t \lambda_{\nu}^{b}}
\end{aligned}
$$

We note that

$$
\zeta_{a, b}\left(f_{1}, f_{2}, P_{\mathcal{B}}\right)(s)=\zeta\left(f_{1}, f_{2}, P_{\mathcal{B}}\right)(b s-a)
$$


the first assertion now follows easily from the representation of the heat content in terms of the residues of the zeta function. Let $\tilde{s}=b s-a$, then

$$
\begin{aligned}
\beta_{n, a, b}\left(f_{1}, f_{2}, P, \mathcal{B}\right) & =\operatorname{Res}_{s=-\frac{n-a d}{b d}} \Gamma(s) \zeta\left(f_{1}, f_{2}, P_{\mathcal{B}}\right)(b s-a) \\
& =\operatorname{Res}_{\tilde{s}=\frac{-n}{d}} \frac{1}{b} \Gamma\left(\frac{\tilde{s}+a}{b}\right) \zeta\left(f_{1}, f_{2}, P_{\mathcal{B}}\right)(\tilde{s}) \\
& =\left.\frac{\Gamma\left(\frac{\tilde{s}+a}{b}\right)}{b \Gamma(\tilde{s})}\right|_{\tilde{s}=\frac{-n}{d}} \operatorname{Res}_{\tilde{s}=-\frac{n}{d}} \Gamma(\tilde{s}) \zeta\left(f_{1}, f_{2}, P_{\mathcal{B}}\right)(\tilde{s}) .
\end{aligned}
$$

This completes the proof if $P_{\mathcal{B}}$ is positive definite. Note that

$$
\beta_{n, a, b}\left(f_{1}, f_{2}, P+c, \mathcal{B}^{b}\right) \quad \text { and } \quad C_{n, d, a, b} \beta_{n}\left(f_{1}, f_{2}, P+c, \mathcal{B}\right)
$$

are polynomial in $c$. They agree for $c$ sufficiently large, since $P_{\mathcal{B}}+c$ will be positive definite. Consequently, these two polynomials agree for all $c$; (b) follows in the general case by setting $c=0$.

\section{Heat content asymptotics on the boundary.}

Let $d y$ denote the Riemannian measure on $\partial M$. We define the heat content on the boundary of $M$ as follows:

$$
b\left(f_{1}, f_{2}, P, \mathcal{B}\right)(t):=\int_{\partial M}\left\langle e^{-t P_{\mathcal{B}}} f_{1}, f_{2}\right\rangle d y .
$$

This invariant monitors the heat distribution on the boundary of $M$, and has a well-defined asymptotic series.

Lemma 3.1. Let $\{P, \mathcal{B}\}$ be admissible. Let $t \rightarrow 0^{+}$. There exists an asymptotic expansion of the form

$$
b\left(f_{1}, f_{2}, P, \mathcal{B}\right)(t) \simeq \sum_{n=0}^{\infty} b_{n}\left(f_{1}, f_{2}, P, \mathcal{B}\right) t^{\frac{n-1}{2}}
$$

where the coefficients $b_{n}$ are locally computable on the boundary.

Proof. Let $i(M)$ be the injectivity radius of $M$. Let $\phi(r) \in C^{\infty}(\mathbb{R})$ be a smooth function which is identically 1 near $r=0$ and vanishes identically near $r=i(M)$. Let $x^{m}$ be the geodesic distance to the boundary, and define

$$
\Psi(x):=\phi\left(x^{m}\right) \in C^{\infty}(M) .
$$


Let $Q=\Psi \nabla_{m}$ on $C^{\infty}(V)$, and let $\widetilde{Q}=\widetilde{\nabla}_{m} \Psi$ on $C^{\infty}\left(V^{*}\right)$. We integrate by parts to see that

$$
\int_{M}\left\{\left\langle Q e^{-t P_{\mathcal{B}}} f_{1}, f_{2}\right\rangle-\left\langle e^{-t P_{\mathcal{B}}} f_{1}, \widetilde{Q} f_{2}\right\rangle\right\} d x=-\int_{\partial M}\left\langle e^{-t P_{\mathcal{B}}} f_{1}, f_{2}\right\rangle d y .
$$

The two expressions on the left-hand side of this equation have well-defined asymptotic expansions, and thus the right-hand side has an asymptotic expansion. Furthermore, this asymptotic expansion can be computed as a boundary integral plus an integral over an arbitrary small collared neighborhood of the boundary. By expanding the $f_{i}$ in a Taylor series in the normal variable we can transform the integral over the collar into the desired boundary integral.

One can now use invariance theory to expand the heat content asymptotics on the boundary for $\{P, \mathcal{B}\}$ in terms of a Weyl basis, and solve for the coefficients using the method of universal examples; for example, see $[\mathbf{D}]$. It turns out that there is a simple relation between these boundary invariants and the heat content invariants. The next two results illustrate that relationship.

Let $T_{1}=a^{\nu} \nabla_{\nu}+b$ be an arbitrary first-order differential operator for some endomorphisms $a^{\nu}$, and $b$ on $C^{\infty}(V)$. Consider the following heat content invariant:

$$
\beta\left(f_{1}, f_{2}, T_{1}, P, \mathcal{B}\right)(t)=\int_{M}\left\langle T_{1} e^{-t P_{\mathcal{B}}} f_{1}, f_{2}\right\rangle d x .
$$

Integration by parts yields

$$
\int_{M}\left\langle T_{1} e^{-t P_{\mathcal{B}}} f_{1}, f_{2}\right\rangle d x=\int_{M}\left\langle e^{-t P_{\mathcal{B}}} f_{1}, \widetilde{T}_{1} f_{2}\right\rangle d x-\int_{\partial M}\left\langle e^{-t P_{\mathcal{B}}} f_{1}, \tilde{a}_{m} f_{2}\right\rangle d y .
$$

Each of these expressions has a well-defined asymptotic expansion. We equate the terms in these expansions to prove:

Lemma 3.2. Let $\{P, \mathcal{B}\}$ be admissible and let $T_{1}=a^{\nu} \nabla_{\nu}+b$ be an arbitrary first-order differential operator. Then

$$
\beta_{n}\left(f_{1}, f_{2}, T_{1}, P, \mathcal{B}\right)=\beta_{n-1}\left(f_{1}, \widetilde{T}_{1} f_{2}, P, \mathcal{B}\right)-b_{n}\left(f_{1}, \tilde{a}_{m} f_{2}, P, \mathcal{B}\right) .
$$

Next, consider an arbitrary second order differential operator $T_{2}$. Assume that $\{P, \mathcal{B}\}$ is admissible and that the boundary operator $\mathcal{B}$ is of Neumann type. In particular, we want

$$
\mathcal{B}_{S}^{+}\left(e^{-t P_{\mathcal{B}}} f_{1}\right)=\left.\left\{\left(e^{-t P_{\mathcal{B}}} f_{1}\right)_{; m}+S\left(e^{-t P_{\mathcal{B}}} f_{1}\right)\right\}\right|_{\partial M}=0,
$$


for some $S \in C^{\infty}\left(\operatorname{End}\left(\left.V\right|_{\partial M}\right)\right)$. Again, integration by parts gives a formula for the asymptotics of $\beta\left(f_{1}, f_{2}, T_{2}, P, \mathcal{B}\right)$ in terms of the asymptotics of the heat content invariants and the boundary invariants.

Lemma 3.3. We adopt the notation established above. If $\mathcal{B}_{S}^{+}\left(e^{-t P_{\mathcal{B}}} f_{1}\right)=0$, then

$$
\beta_{n}\left(f_{1}, f_{2}, T_{2}, P, \mathcal{B}\right)=\beta_{n-2}\left(f_{1}, \widetilde{T}_{2} f_{2}, P, \mathcal{B}\right)+b_{n-1}\left(f_{1}, \mathcal{R} f_{2}, P, \mathcal{B}\right),
$$

for some differential operator $\mathcal{R}$.

It is interesting to note that, since the asymptotics for the standard and the generalized heat content differ by a boundary term, any additional information contained in the generalized heat content is captured by the boundary invariant. As a result, the task of computing the asymptotics for a generalized heat operator is reduced to that of computing a related boundary invariant, which is often a simpler calculation, and of considering the asymptotics for the standard heat operator, many of which are already known.

We now compute the boundary asymptotics associated with an operator of Laplace type. Recall that an operator $P=D$ is said to be of Laplace type whenever there exist both a unique connection $\nabla$ and a unique endomorphism $E$ such that we can express $D$ in the form

$$
D=-\left(g^{\nu \mu} \nabla_{\nu} \nabla_{\mu}+E\right) .
$$

Since $\partial M \neq \emptyset$, we must impose suitable boundary conditions. Let $S$ be an auxiliary endomorphism of $\left.V\right|_{\partial M}$, and let $\nabla_{m}$ denote the normal covariant derivative with respect to the inward unit normal. The Dirichlet $\left(\mathcal{B}^{-}\right)$and Neumann $\left(\mathcal{B}_{S}^{+}\right)$boundary operators are defined by

$$
\mathcal{B}^{-} f:=\left.f\right|_{\partial M} \quad \text { and } \quad \mathcal{B}_{S}^{+} f:=\left.\left(\nabla_{m}+S\right) f\right|_{\partial M} .
$$

Dirichlet and Neumann boundary conditions are adequate for most physical applications. However, in certain field theories or when studying elliptic complexes, it is often necessary to impose a mixture of Dirichlet and Neumann boundary conditions. Assume that, near the boundary of $M$, we are giving a splitting $V=V_{-} \oplus V_{+}$. Let $\Pi_{ \pm}$be the associated projections on $V_{ \pm}$. We define the mixed boundary operator by imposing Dirichlet boundary conditions on $V_{-}$and Neumann boundary conditions on $V_{+}$.

We start by considering the case where $\mathcal{B}=\mathcal{B}^{-}$is a Dirichlet boundary operator. The heat operator satisfies the boundary conditions; therefore, we have $\left.\left(e^{-t D_{\mathcal{B}}} f_{1}\right)\right|_{\partial M}=0$, and it follows that

$$
\int_{\partial M}\left\langle e^{-t D_{\mathcal{B}}} f_{1}, f_{2}\right\rangle d y=0 .
$$


Therefore, $b_{n}\left(f_{1}, f_{2}, D, \mathcal{B}^{-}\right)=0$ for all $n \geq 0$, as expected.

Next, let $\mathcal{B}=\mathcal{B}_{S}^{+}$define a boundary operator of Neumann type. Substituting $\left\{D, \mathcal{B}_{S}^{+}\right\}$in Lemma 3.3 yields

$$
b_{n-1}\left(f_{1}, \widetilde{\mathcal{B}}_{S}^{+} f_{2}, D, \mathcal{B}_{S}^{+}\right)=\beta_{n-2}\left(f_{1}, \widetilde{D} f_{2}, D, \mathcal{B}_{S}^{+}\right)-\beta_{n}\left(f_{1}, f_{2}, D, D, \mathcal{B}_{S}^{+}\right) .
$$

We use Lemma 2.1 with $a=b=1$ to write

$$
b_{n-1}\left(f_{1}, \widetilde{\mathcal{B}}_{S}^{+} f_{2}, D, \mathcal{B}_{S}^{+}\right)=\beta_{n-2}\left(f_{1}, \widetilde{D} f_{2}, D, \mathcal{B}_{S}^{+}\right)+\frac{n}{2} \beta_{n}\left(f_{1}, f_{2}, D, \mathcal{B}_{S}^{+}\right) .
$$

Since the heat content asymptotics are known to order $n=6$ for a Laplace operator with Neumann boundary conditions [DG], we can use the last identity to compute the following asymptotics.

Theorem 3.4. Let $D$ be an operator of Laplace type and let $\mathcal{B}=\mathcal{B}_{S}^{+}$define Neumann boundary conditions. Then

(a) $b_{0}\left(f_{1}, f_{2}, D, \mathcal{B}_{S}^{+}\right)=0$.

(b) $b_{1}\left(f_{1}, f_{2}, D, \mathcal{B}_{S}^{+}\right)=\int_{\partial M}\left\langle f_{1}, f_{2}\right\rangle d y$.

(c) $b_{2}\left(f_{1}, f_{2}, D, \mathcal{B}_{S}^{+}\right)=2 \pi^{-1 / 2} \int_{\partial M}\left\langle\mathcal{B}_{S}^{+} f_{1}, f_{2}\right\rangle d y$.

(d) $b_{3}\left(f_{1}, f_{2}, D, \mathcal{B}_{S}^{+}\right)=\int_{\partial M}\left\{\left\langle S \mathcal{B}_{S}^{+} f_{1}, f_{2}\right\rangle+\frac{1}{2} L_{a a}\left\langle\mathcal{B}_{S}^{+} f_{1}, f_{2}\right\rangle\right.$ $\left.+\left\langle\left(f_{1 ; m m}+f_{1: a a}-L_{a a} f_{1 ; m}+E f_{1}\right), f_{2}\right\rangle\right\} d y$.

(e) $b_{4}\left(f_{1}, f_{2}, D, \mathcal{B}_{S}^{+}\right)=-\frac{2}{3} \pi^{-1 / 2} \int_{\partial M}\left\{\left\langle\mathcal{B}_{S}^{+} D f_{1}, f_{2}\right\rangle+\left\langle\left(\mathcal{B}_{S}^{+} f_{1}\right)_{: a}, f_{2: a}\right\rangle\right.$ $\left.-\left\langle\left(E+2 S^{2}+2 S L_{a a}+\frac{1}{4} L_{a a} L_{b b}+\frac{1}{2} L_{a b} L_{a b}-\frac{1}{2} R_{a m a m}\right) \mathcal{B}_{S}^{+} f_{1}, f_{2}\right\rangle\right\} d y$.

Remark. We use the following notational conventions. Near the boundary, we choose coordinates $\left(y, x^{m}\right)$, where $y=\left(y^{1}, \cdots, y^{m-1}\right)$ is a system of local coordinates on $\partial M$ and $x^{m}$ is the geodesic distance to the boundary. We choose an orthonormal frame so that $e_{m}=\partial_{m}$. Roman indices $a, b, \ldots$ index orthonormal frames for the tangent and cotangent bundles of the boundary and range from 1 through $m-1$. Let $\nabla$ and $\nabla^{\partial M}$ be the Levi-Civita connection on $M$ and on the boundary respectively. We denote by ";" multiple covariant differentiation with respect to $\nabla$, and denote by ":" multiple tangential covariant differentiation with respect to $\nabla^{\partial M}$ on the boundary. Note that if $f \in C^{\infty}(M)$, then $f_{; a}=f_{: a}$ on $\partial M$, since there are no tangential indices to be differentiated. On the other hand, $f_{; a b} \neq f_{: a b}$ as the index $a$ is also being differentiated. Let $f_{; m}$ be the covariant derivative of $f$ with respect to the inward pointing unit normal. Then $f_{; a b}=f_{: a b}-L_{a b} f_{; m}$. The second fundamental form $L_{a, b}$ is defined on the boundary of $M$ and measures the difference between $\nabla$ and $\nabla^{\partial M}$.

\section{Asymptotics for operators of Dirac type.}

Let $\operatorname{End}(V)$ denote the bundle of endomorphisms of $V$. A Clifford module structure on $V$ is a linear map $\gamma: \operatorname{Cliff}(T M) \rightarrow \operatorname{End}(V)$ so $\gamma(\xi)^{2}=-|\xi|^{2} \cdot I_{V}$. 
A first-order differential operator $Q$ on $C^{\infty}(V)$ is called an operator of Dirac type if the leading symbol of $Q$ defines a Clifford module structure on $V$, or equivalently if $Q^{2}$ defines an operator of Laplace type.

Let $\gamma^{\nu}=\gamma\left(d x^{\nu}\right)$. Locally, we can write

$$
Q=\gamma^{\nu} \partial_{\nu}-r
$$

where $r$ is a local section of $\operatorname{End}(V)$, and $\gamma$ satisfies the Clifford commutation relations:

$$
\gamma^{\nu} \gamma^{\mu}+\gamma^{\mu} \gamma^{\nu}=-2 g^{\nu \mu} \cdot I_{V}
$$

Let $Q$ be an operator of Dirac type on $C^{\infty}(V)$, and let $D=Q^{2}$ be the associated operator of Laplace type. Since $\partial M \neq \emptyset$, we must impose suitable boundary conditions. There are natural boundary operators for $Q$ and $D$; they are determined by the Clifford module structure on $V$.

Definition. We say that $\{Q, \chi\}$ is admissible if

(i) $Q: C^{\infty}(V) \rightarrow C^{\infty}(V)$ is a first order partial differential operator of Dirac type.

(ii) $\chi \in C^{\infty}(\operatorname{End}(V))$ is defined on $\partial M$ and satisfies $\chi^{2}=1, \chi \gamma_{m}+\gamma_{m} \chi=$ 0 , and $\chi \gamma_{a}=\gamma_{a} \chi$ for $a \leq m-1$.

For example, let $V=\Lambda(M)$ and let $Q=d+\delta$ be the de Rham operator. Near the boundary, we decompose

$$
\Lambda(M)=e_{m} \wedge \Lambda(\partial M) \oplus \Lambda(\partial M)
$$

Let $\chi=I \oplus-I$. It is easily verified that $\{Q, \chi\}$ is admissible. The endomorphisms $\pm \chi$ define absolute and relative boundary conditions respectively.

We use the endomorphism $\chi$ to define a boundary operator $\mathcal{B}$. Assume that $\{Q, \chi\}$ is admissible. Using parallel transport along normal geodesic rays, we can extend $\chi$ to a neighborhood of $\partial M$, so that near $\partial M$ we have

$$
\chi_{; m}=0, \quad \chi^{2}=1, \quad \text { and } \quad \chi \gamma_{m}+\gamma_{m} \chi=0 .
$$

This defines a splitting $\Pi_{ \pm}:=\frac{1}{2}(1 \pm \chi)$ for $V=V_{-} \oplus V_{+}$. We define boundary operators for $Q$ and $D$ relative to this decomposition by

$$
\mathcal{B}_{\chi} f=\left.\Pi_{-} f\right|_{\partial M}
$$

and

$$
\mathcal{B}_{\chi}^{2} f=\left.\left.\Pi_{-} f\right|_{\partial M} \oplus \Pi_{-} Q f\right|_{\partial M} .
$$


The boundary conditions determined by $\mathcal{B}_{\chi}^{2}$ can also be obtained from a boundary operator of mixed type. We refer to $([\mathrm{BrG}]$, Lemma 7$)$ for the proof of:

Lemma 4.1. Define $\Phi \in C^{\infty}(\operatorname{End}(V))$ by $Q=\gamma^{\nu} \nabla_{\nu}^{D}-\Phi$. Define

(i) $\mathcal{B} f=\left.\left.\Pi_{-} f\right|_{\partial M} \oplus \Pi_{+}\left(\nabla_{m}+\gamma_{m} \Phi-\frac{1}{2} \gamma_{m} \gamma_{a} \chi_{; a}\right) \Pi_{+} f\right|_{\partial M}$.

(ii) $\mathcal{B}_{\chi}^{2} f=\left.\left.\Pi_{-} f\right|_{\partial M} \oplus \Pi_{-} Q f\right|_{\partial M}$.

Then $\mathcal{B} f=0$ if and only if $\mathcal{B}_{\chi}^{2} f=0$.

Remark. The boundary operator $\mathcal{B}$ is a boundary operator of mixed type with $S \in C^{\infty}(\operatorname{End}(V))$ given by $S=\Pi_{+}\left(\gamma_{m} \Phi-\frac{1}{2} \gamma_{m} \gamma_{a} \chi_{: a}\right) \Pi_{+}$. This equivalence of the boundary conditions guarantees the existence of an asymptotic expansion for $\beta\left(f_{1}, f_{2}, Q, Q^{2}, \mathcal{B}_{\chi}^{2}\right)$.

We study the following heat equation invariant:

$$
\beta\left(f_{1}, f_{2}, Q, Q^{2}, \mathcal{B}_{\chi}^{2}\right)(t):=\int_{M}\left\langle Q e^{-t Q_{\mathcal{B}}^{2}} f_{1}, f_{2}\right\rangle d x .
$$

We write $\beta\left(f_{1}, f_{2}, Q, \chi\right)$ instead of $\beta\left(f_{1}, f_{2}, Q, Q^{2}, \mathcal{B}_{\chi}^{2}\right)$ to simplify notation. There exists an asymptotic expansion

$$
\beta\left(f_{1}, f_{2}, Q, \chi\right)(t) \simeq \sum_{n=0}^{\infty} \beta_{n}\left(f_{1}, f_{2}, Q, \chi\right) t^{\frac{n-1}{2}} .
$$

We use Lemma 3.2 to compute these asymptotics. Let $D=Q^{2}$, and let $\mathcal{B}$ be the mixed boundary operator determined by $\chi$. If we let $\check{f}_{2}=-\gamma_{m} f_{2}$, then

$$
\beta_{n}\left(f_{1}, \check{f}_{2}, Q, \chi\right)=\beta_{n-1}\left(f_{1}, Q \check{f}_{2}, D, \mathcal{B}\right)+b_{n}\left(f_{1}, f_{2}, D, \mathcal{B}\right) .
$$

The asymptotics for an operator of Laplace type with mixed boundary conditions for $n=0,1,2$, and 3 have been computed in [DG, Theorem 4.2]. Thus the $\beta_{n}\left(f_{1}, Q \check{f}_{2}, D, \mathcal{B}\right)$ are known. The boundary asymptotics for an operator of Laplace type have been computed in Section 3 for Dirichlet and Neumann boundary conditions. In general, the projections $\Pi_{ \pm}$may fail to commute with the endomorphism $\Phi$ and the connection $\nabla$, giving rise to extra terms in the expansion. Invariants measuring this failure have order at least 2 and do not enter into $b_{n}$ for $n \leq 2$. To obtain the lower order asymptotics for mixed boundary conditions, it will therefore suffice to combine the terms arising in the asymptotics for Dirichlet and Neumann boundary conditions. We use Theorem 3.4 to obtain

\section{Theorem 4.2.}

(a) $\beta_{0}\left(f_{1}, f_{2}, Q, \chi\right)=0$. 
(b) $\beta_{1}\left(f_{1}, f_{2}, Q, \chi\right)=\int_{M}\left\langle Q f_{1}, f_{2}\right\rangle d x+\int_{\partial M}\left\langle\gamma_{m} f_{1}, f_{2 ; m}^{+}\right\rangle d y$.

(c) $\beta_{2}\left(f_{1}, f_{2}, Q, \chi\right)=2 \pi^{-\frac{1}{2}} \int_{\partial M}\left\{\left\langle f_{1 ; m}^{+}, \gamma_{m} f_{2}^{-}\right\rangle+\left\langle\gamma_{m} f_{1}^{-}, f_{2 ; m}^{+}\right\rangle+\left\langle S f_{1}^{+}, \gamma_{m} f_{2}^{-}\right\rangle\right.$ $\left.+\left\langle\gamma_{a} f_{1}^{-}, f_{2: a}\right\rangle+\left\langle\Phi f_{1}^{-}, f_{2}\right\rangle\right\} d y$.

Acknowledgments. Parts of these results appear in my doctoral dissertation. I am grateful to Peter Gilkey for suggesting the problem, and for several helpful conversations concerning this work.

\section{References}

[APS] M.F. Atiyah, R. Bott and I.M. Singer, Spectral asymmetry and riemannian geometry, I, Math. Proc. Cambridge Philos. Soc., 77 (1975), 43-69.

[BD] M. van den Berg and E.B. Davies, Heat flow out of regions in $\mathbb{R}^{m}$, Math. Z., 202 (1989), 463-482.

[BG] M. van den Berg and P. Gilkey, Heat content asymptotics of riemannian manifold with boundary, J. Funct. Anal., 120 (1994), 48-71.

[BrG] T.P. Branson and P. Gilkey, Residues of the eta function for an operator of Dirac type with local boundary conditions, Diff. Geom. and its Appl., 2 (1992), 249-267.

[D] S. Desjardins, Heat content asymptotics, Doctoral Dissertation, University of Oregon, 1995.

[DG] S. Desjardins and P. Gilkey, Heat content asymptotics for operators of Laplace type with Neumann boundary conditions, Math. Z., 215 (1994), 251-268.

[FG] H. Fegan and P. Gilkey, Invariants of the heat equation, Pac. J. of Math., 117(2) (1985), 233-254.

[McA] M. McAvity, Surface energy from heat content asymptotics, J. of Phys, A26 (1993), 823-830.

[MP] S. Minakshisundaram and A. Pleijel, Some properties of the eigenfunction of the Laplace operator on Riemannian manifolds, Can. J. Math., 1 (1949), 242-256.

Received September 20, 1996. This research was partially supported by the CMS and NSERC.

UNIVERSITY OF OTTAWA

OTTAWA, ONTARIO

CANADA K1N 6N5

E-mail address: desjard@uottawa.ca

Note: THERE WERE INCORRECT REFERENCES IN THE PAPER VERSION IN THE SENTENCE JUST PRIOR TO THEOREM 4.2. 